\title{
A second type of rod opsin cDNA from the common carp (Cyprinus carpio) ${ }^{1}$
}

\author{
Jormay Lim, Jean-Leon Chang, Huai-Jen Tsai * \\ Institute of Fisheries Science, National Taiwan University, Taipei 106, Taiwan \\ Received 11 February 1997; accepted 20 February 1997
}

\begin{abstract}
A second type of rhodopsin cDNA from carp (cRh-II) shared $97.2 \%$ polynucleotide identity with the previously reported cRh-I. The deduced amino acid sequences of cRh-I and cRh-II exhibited 98.6\% identity. The key difference between these two types of $\mathrm{cRh}$ is that valine at position 169 of $\mathrm{cRh}-\mathrm{I}$ was replaced by glutamic acid in cRh-II. Southern blot analysis of genomic DNA showed that there were two types of $\mathrm{cRh}$ gene. These two rod opsin genes were proven to be expressed in carp retinas by using RT-PCR with type-specific primers. (c) 1997 Elsevier Science B.V. All rights reserved.
\end{abstract}

Keywords: Photoreceptor; Retinal cDNA library; Transmembrane domain; Visual pigment; (Fish)

The underwater environment limits both the intensity and the spectral bandwidth of ambient light for vision [1]. Although the retinal anatomy and photoreceptor proteins (opsins) of land and aquatic vertebrates show structural similarities [2,3], it has been proposed that fish have a tetrachromatic visual system, in contrast to the trichromatic system in humans, consisting of four types of cone opsins [4,5]. A blue, a red and two green cone opsin genes were isolated from goldfish [6]. Each type of cone opsin can be readily distinguished by the wavelengths of light each absorbs maximally. Rod opsin, rhodopsin, is another photoreceptor but locates at rod cell, which mediates dim-light vision. In order to further understand comparative aquatic visual physiology, we studied the

\footnotetext{
* Corresponding author. Fax: +886 2 3638483; E-mail: hjtsai@ccms.ntu.edu.tw

${ }^{1}$ This sequence has been submitted to the EMBL Nucleotide Sequence Database with accession No. Z71999.
}

opsins of the common carp (Cyprinus carpio), a bottom-dwelling species important in aquaculture.

Of the seven-membrane-spanning photoreceptor proteins, rhodopsin is the one most completely investigated in terms of the relationship between its structure and function [7,8]. After bovine and human rhodopsin cDNA were first reported by Nathans and Hogness $[9,10]$, the rhodopsin cDNA of many species was cloned including Drosophila melanogaster [11], chickens [12], mice [13], frogs [14] and squid [15]. Full-length cDNAs encoding fish rhodopsin were also reported for lampreys [16], sand gobies [17], goldfish [6] and common carp [18]. In addition, partial nucleotide sequences of the rhodopsin gene in eight fish species from the Euteleostei and the Elopomorpha were amplified by PCR [19]. Only one type of rhodopsin gene was found in each species studied. However, the common carp possesses a second type of rhodopsin (cRh-II) cDNA, that is not totally identical to the one (cRh-I) we reported previously [18]. This is the first time two rhodopsin cDNAs, which 
are not non-functional processed pseudogenes, have been found in one species.

A positive clone was isolated from carp retinal cDNA library [18] using goldfish rhodopsin cDNA as a probe. This putative clone contained an insert DNA of around $1.7 \mathrm{~kb}$, which was longer than the previous $1.6 \mathrm{~kb}$ cRh-I cDNA clone [18]. Unlike the cRh-I cDNA fragment, this insert cDNA (cRh-II) could not be digested with SmaI but could be digested with Pst I. Two DNA fragments of 0.04 and $1.59 \mathrm{~kb}$ were generated after PstI restriction.

The dideoxynucleotide chain-termination method [20] was used to sequence cRh-II. In addition to T7 and T3 primers, seven primers were synthesized for determining the DNA sequences of both strands. For sense strand reading, primers CRH1 (5'-CCTGGCCGCGTACATGTTC-3', nt 188-206), CRH3 (5'GGGGGTTGTCTTCACC-3', nt 536-551) and R1 (5'-GGATCCCATATGCCAGCGTG-3', nt 865-884) were used. For antisense strand reading, primers L2 (5'-GGTTGCAGCCGACGCGTCCA-3', nt 405386), CRH4 (5'-CACAGGGTGGTGATCATGC-3', nt 1036-1017), CRH2 (5'-GGGACATGGTCTGTC3', nt 1345-1331), and CRH5 (5'-GGAGTCCAACAGTAAGAGAG-3', nt 1469-1450) were used.

The polynucleotide sequences of cRh-I and $\mathrm{cRh}$-II, which shared $97.2 \%$ identity, are shown in Fig. 1. cRh-I cDNA and cRh-II cDNA (1) consist of a single open reading frame of 1062 nucleotides (nt), located between nt positions 72 and 1133, that encodes 354 amino acid (aa) residues; (2) have a translation initiation codon (ATG) at nt positions $72-74$, preceded by 71 untranslated leader $\mathrm{nt}$; and (3) have a translation termination codon TAA, at nt positions 1134-1136, followed by an $3^{\prime}$ untranslated region. However, cRh-II had 495 untranslated nt at the $3^{\prime}$ end, while cRh-I had only 397 untranslated nt [18].

There was only one polyadenylation signal in cRh-I cDNA, which started at nt position $1511,15 \mathrm{nt}$ upstream from the start of the 52 A tail [18]. In contrast, cRh-II cDNA had four polyadenylation signals (AATAAA or AATTAAA) at the $3^{\prime}$ end of the untranslated region. The four signals started at nt positions 1254, 1521, 1531 and 1611, respectively. The signal starting at position 1521 of $\mathrm{cRh}-\mathrm{II} \mathrm{cDNA}$ was identical to the signal located at position 1511 of cRh-I cDNA. The last polyadenylation signal for cRh-II cDNA, located at position 1611, started $14 \mathrm{nt}$ upstream from the start of the 30 A tail. cRh-II cDNA was 99 bp longer than cRh-I. This 99 bp DNA segment was located at the $3^{\prime}$ and is specific for carp cRh-II cDNA. Thus, cRh-I cDNA is not one of the alternative transcription products of the $\mathrm{cRh}$-II gene.

The deduced aa sequences of type I and type II carp rhodopsin differed in only 5 out of 354 residues and exhibited $98.6 \%$ identity. cRh-II rhodopsin, like cRh-I, contained all the common residues present in the rhodopsins of species including humans [10], mice [13], chickens [12], lampreys [16], goldfish [6] and sand gobies [17]. Conserved aa included a largely hydrophobic polypeptide with a predominantly hydrophobic N-terminal, glycosylation sites (Asn-2 and Asn-15), disulfide bond formation residues (between Cys-110 and Cys-187), formation sites for Schiffbase counterions (between Glu-113 and Lys-296), interaction sites for the G-protein transducin (Glu-134 and Arg-135), the control ling site of the equilibrium between photo-activated metarhodop sin I and metarhodopsin II (His-211), the palmitoylation sites essential to membrane anchoring (Cys-322 and Cys323), and the phosphorylation control sites (Ser/Thr segment at C-terminal loop).

Of the five aa residues of $\mathrm{cRh}$-II that differed from those of cRh-I [18], two were non-homologous alterations (Val-169 and His-315 of cRh-I were replaced by Glu-169 and Asn-315 of cRh-II, respectively) while three were homologous replacements for residues having similar properties (Val-19 $\rightarrow$ Ile-19; Ile-54 $\rightarrow$ Val-54 and Ile-108 $\rightarrow$ Val-108).

A Chargpro program was used to estimate the $\mathrm{p} I$ of cRh-II. A p I value of 5.9 was obtained for the predicted $\mathrm{cRh}-\mathrm{II}$ in contrast to the $6.3 \mathrm{p} I$ value of cRh-I [18]. The $\mathrm{p} I$ values for both types of carp rhodopsins are in the 5.9-6.3 range observed for bovine and human rhodopsins. However, these $\mathrm{p} I$ values differ markedly from the alkaline $\mathrm{p} I$ values of approximately 9.5 observed for color and photopic cone pigments [21].

Hydropathicity profiles of rhodopsin aa sequences were analyzed by Rao and Argos [22], using the RAOARGOS program from the PC/GENE software package. The most common lengths of helix IV are 20-21 aa. Helix IV consists of 21 residues in human [10], mouse [13], bovine [9], chicken [12] and carp type I rhodopsins [18] and 20 residues in lamprey [16] and fruit fly rhodopsins [11]. However, the length 
R1 ACACAACATCCAACCGCAGCCATGAACGGTACAGAGGGACCTATGTTCTA 100

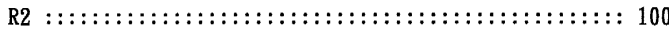
R1 II N G $T$ T E $G$ P

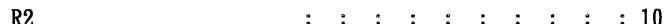

R1 CGTGCCTATGTCCAATGCGACCGGCGTTGTTAAGAGCCCGTACGACTATC 150

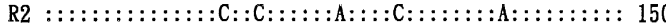

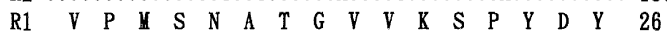
R2 : : : : : : : : : : $:$ I $\quad: \quad: \quad: \quad: \quad: \quad: \quad: \quad 26$

R1 CCCAGTACTACCTGGTGGCGCCATGGGCATACGGCTGCCTGGCCGCGTAC 200

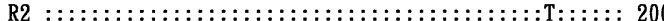
$\begin{array}{lllllllllllllllllll}R 1 & P & Q & Y & Y & L & V & A & P & \mathbb{N} & \text { A } & Y & G & \text { C } & \text { L } & \text { A } & \text { A } & Y & 43\end{array}$ R2 : : : : : : : : : $:$ : $:$ : $:$ : $:$ : $\quad: \quad$ : $\quad$ : 43

R1 ATGTTCTTCCTCATTATCACTGGCTTCCCTATCAACTTCCTCACTCTGTA 250

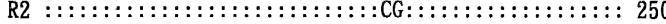

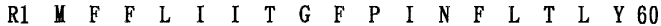
$\mathrm{R} 2$ : : : : : : : : : : V : : : : : $: 60$

R1 CGTCACCATCGAGCACAAGAAGCTGCGTACACCTCTCAACTACATTCTGC 300

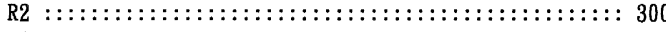
$\begin{array}{llllllllllllllllll}\text { R1 } & \text { V } & \text { T } & \text { I } & \text { E } & H & \text { K } & \text { K } & \text { L } & \text { R } & \text { T } & \text { P } & \text { L } & \text { N } & \text { Y } & \text { I } & \text { L } & 76\end{array}$

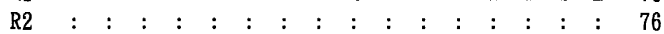

R1 TGAACCTCGCCATTTCCGACCTCTTCATGGTGTTCGGTGGCTTCACCACG 350

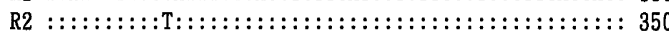
$\begin{array}{lllllllllllllllllll}\text { R1 L } & \text { N } & \text { L } & \text { A } & \text { I } & \text { S } & \text { D } & \text { L } & \text { F } & \text { HI } & \text { V } & \text { F } & \text { G } & \text { G } & \text { F } & \text { T } & \text { T } & 93\end{array}$

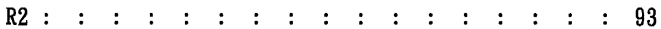

R1 ACGATGTACACGTCGTTGCATGGCTACTTTGTTTTTGGACGCATTGGCTG 400

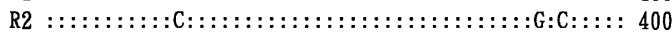
$\begin{array}{llllllllllllllllllll}R & \text { T } & Y & Y & \text { T } & S & \text { L } & H & G & Y & F & V & F & G & R & I & G & C & 110\end{array}$ R2 $: \quad: \quad: \quad: \quad: \quad: \quad: \quad: \quad: \quad: \quad: \quad: \quad: \quad: \quad \mathrm{V} \quad: \quad: 110$ R1 CAACCTCGAAGGCTTCTTCGCAACCCTGGGTGGTGAAATGGGCCTTTGGT 450

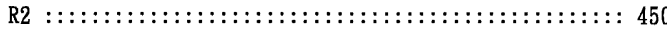

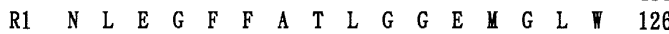

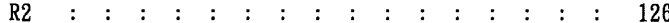

R1 CCTTGGTGGTGCTGGCCTTCGAGAGGTGGATGGTTGTCTGTAAGCCCGTG 500

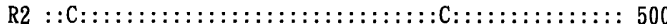
R1 S L L V V V $\mathrm{R} 2 ： \quad: \quad: \quad: \quad: \quad: \quad: \quad: \quad: \quad: \quad: \quad: \quad: \quad: \quad: \quad: \quad: 143$

R1 AGCAACTTCCGCTTCGGAGAGAACCACGCCATCATGGGGGTTGTCTTCAC 550

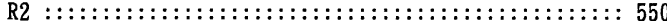
$\begin{array}{llllllllllllllllllllll}R & S & N & F & R & F & G & E & N & H & A & I & \text { I } & G & V & V & F & T & 160\end{array}$

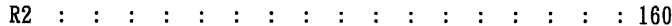

R1 CTGGTTCATGGCCTGCACCTGCGCCGTGCCTCCCCTGGTCGGCTGGTCCC 600

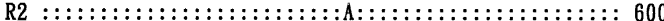

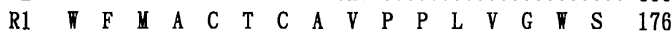

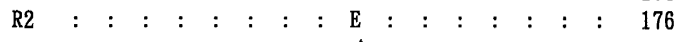
$\boldsymbol{A}$

R1 GTTACATCCCCGAGGGCATGCAGTGCTCGTGCGGAGTCGACTATTACACT 650

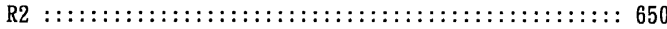
$\begin{array}{lllllllllllllllllll}\text { R1 R } & Y & \text { I } & \text { P } & \text { E } & G & \text { If } & \text { Q } & \text { C } & \text { S } & C & G & \text { V } & \text { D } & \text { Y } & \text { Y } & \text { T } & 193\end{array}$

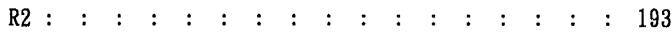

R1 CGCGCCCCTGGCTACAACAATGAGTCCTTTGTCATCTACATGTTCCTTGT 700

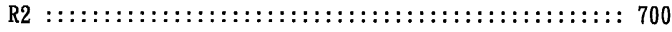
$\begin{array}{lllllllllllllllllll}\text { R1 } & R & \text { A } & \text { P } & G & Y & \text { N } & \text { N } & \text { E } & \text { S } & \text { F } & \text { V } & \text { I } & \text { Y } & \text { I } & \text { F } & \text { L } & \text { V } & 210\end{array}$ R2 : : : : : : : : : : : $:$ : $:$ : $\quad$ : $\quad 210$

R1 CCACTTCATTATTCCATTAATCGTCATATTCTTCTGCTACGGCCGTCTCG 750

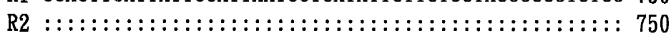
R1 $\begin{array}{lllllllllllllllll}\text { H } & \text { F } & \text { I } & \text { I } & \text { P } & \text { L } & \text { I } & \text { V } & \text { I } & \text { F } & \text { F } & \text { C } & \text { Y } & \text { G } & \text { R } & \text { L } & 227\end{array}$ R2 : : : : : $\quad: \quad: \quad: \quad: \quad: \quad: \quad: \quad: \quad: \quad: \quad: \quad: \quad 227$
R1 TCTGCACCGTCAAAGATGCCGCTGCCCAGCAGCAGGAGTCTGAGACCACC 800

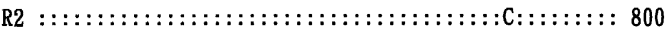
$\begin{array}{lllllllllllllllllll}\text { R1 V } & C & \text { T } & \text { V } & K & \text { D } & \text { A } & \text { A } & \text { A } & Q & Q & Q & \text { E } & \text { S } & \text { E } & \text { T } & \text { T } & 243\end{array}$ $\mathrm{R} 2: \quad: \quad: \quad: \quad: \quad: \quad: \quad: \quad: \quad: \quad: \quad: \quad: \quad: \quad: \quad: \quad 243$

R1 CAGAGGGCTGAGCGTGAGGTCACCCGCATGGTCGTCATCATGGTCATCGG 850

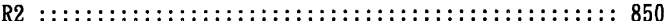

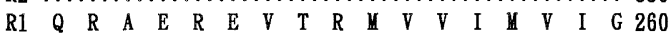
$\mathrm{R} 2::: \quad: \quad: \quad: \quad: \quad: \quad: \quad: \quad: \quad: \quad: 260$

R1 CTTCTTGATTTGCTGGATCCCATATGCCAGCGTGGCCTGGTATATCTTCA 900

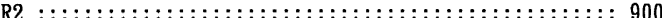

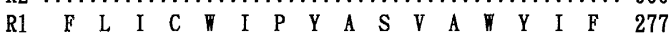
R2 : : : : : : : : : : : $: \quad: \quad: \quad \leq 277$

R1 CCCACCAGGGAAGCGAATTTGGGCCTGTCTTCATGACCGTGCCAGCCTTC 950

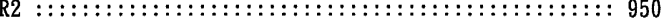
$\begin{array}{lllllllllllllllllll}R 1 & T & H & Q & G & S & E & F & G & P & V & F & \text { I } & T & V & P & A & F & 293\end{array}$ R2 : : : : : : : : : : : : : : : $\quad$ : $\quad$ : $\quad$ : $\quad$ : $\quad$ : 293

R1 TTTGCCAAGAGTGCTGCTGTCTACAACCCATGCATCTACATCTGCATGAA 1000

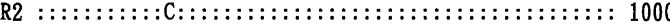
$\begin{array}{lllllllllllllllllll}\text { R1 } & \text { F } & \text { A } & \text { K } & \text { S } & \text { A } & \text { A } & \text { V } & \text { Y } & \text { N } & \text { P } & \text { C } & \text { I } & \text { Y } & \text { I } & \text { C } & \text { II } & \text { N } & 310\end{array}$

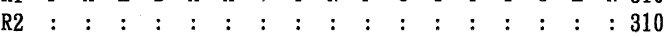

R1 CAAGCAGTTCCGTCACTGCATGATCACCACCCTGTGCTGCGGCAAGAACC 1050

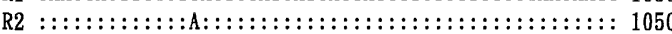
$\begin{array}{llllllllllllllllll}\mathrm{R} 1 & \mathrm{~K} & \mathbf{Q} & \mathrm{F} & \mathrm{R} & \mathrm{H} & \mathrm{C} & \mathbf{H} & \mathrm{I} & \mathrm{T} & \mathrm{T} & \mathrm{L} & \mathrm{C} & \mathrm{C} & \mathrm{G} & \mathrm{K} & \mathrm{N} & 327\end{array}$ $\mathrm{R} 2 \quad: \quad: \quad: \quad: \quad \mathrm{N} \quad: \quad: \quad: \quad: \quad: \quad: \quad: \quad: \quad: \quad: \quad: \quad 327$ R1 CCTTCGAGGAGGAAGAGGGCGCCTCCACTACTGCATCCAAGACCGAGGCT 1100

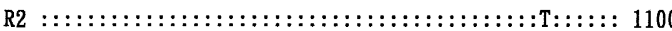
$\begin{array}{lllllllllllllllllll}\text { R1 } & \text { P } & \text { F } & \text { E } & \text { E } & \text { E } & \text { E } & \text { G } & \text { A } & \text { S } & \text { T } & \text { T } & \text { A } & \text { S } & \text { K } & \text { T } & \text { E } & \text { A } & 343\end{array}$

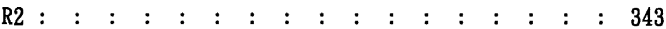
R1 TCGTCCGTGTCTTCCAGCTCCGTGTCCCCTGCGTAAACAGTTGTCCGTGA 1150

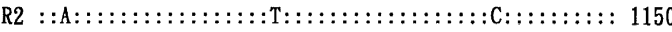
$\begin{array}{llllllllllllll}\text { R1 } & S & S & V & S & S & S & S & V & S & P & A & 354\end{array}$ R2 : : : : : : : $\quad: \quad: \quad: \quad: \quad: \quad: \quad 354$

R1 CACAGAATAAGCAGTGACATGCACTGGGCTTCAACGGCAACCGACGACAC 1200

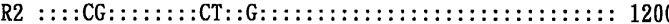

R1 AGAGACCACAAAGTGTTCAGCCCCGGGAAA-GAGCAACCACTACCACTTG 1249

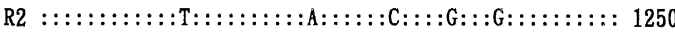

R1 CAGAA-AAAAATGTCTGTCAGTTTTCCTTTTTGTATTTTCACAAAACCCA 1298

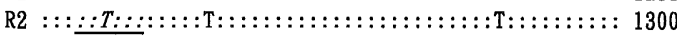

R1 ATTGGTTCAACCAAAAGACAGTTTTGAGAGAGGACAGACCATGTCCCAGT 1348

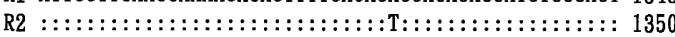

R1 TTCAGTACATCCAGCGAGTCCAGCGTAACGGTGCATAAGATT-------- 1390

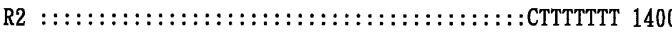

R1 -TTTTTTAATTTTTTCTTCCTAAAATGCAGCAAAAGGAAAAATATCTTAA 1439

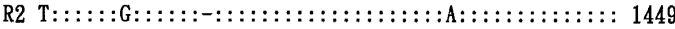

R1 CTCTCTTACTGTTGGACTCCTTATACTGGCTTTGTTGTGATTGTAGAGGC 1489

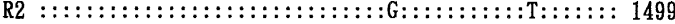

R1 ATGTATTCAAGGCAACGTAACAATAAAAAGCACTTTGCAAATT 1532

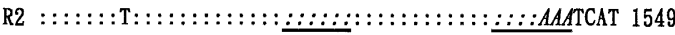

R2 TCTGTTTATGATTTGATTGAGCTGTAATGCTAAAGAAATGTCTAAATAGT 1599 R2 ATTTTAAATTT AATAAACGATACTGATTTCT 1630

Fig. 1. Comparison of the nucleotide sequences and the deduced amino acid sequences of cRh-I (R1) and cRh-II (R2). Identical bases and residues are indicated by double dots (:). Different amino acids are marked with solid triangles. Gaps (-) are created to maximize the degree of homology between the cRh-I and cRh-II sequences. The polyadenylation signals are italicized and underlined. 
of helix IV of cRh-II was only 16 aa. The special structure of helix IV of cRh-II may result from the charged residue (Glu) at position 169 . The residue at position 169 of $\mathrm{cRh}-\mathrm{I}$ and the rhodopsins of other species is the hydrophobic residue, Val.

Genomic DNA was extracted from carp muscle, digested with EcoRI, Pst I, and EcoRI plus Pst I, respectively, and Southern blot analysis was performed with a radio-labelled probe. One positive band, representing a $6.6 \mathrm{~kb}$ fragment from the EcoRI-digested genome, and two positive bands, representing 4 and $2 \mathrm{~kb}$ fragments from both the Pst Idigested genome and the EcoRI- plus PstI-digested genome, were observed (Fig. 2). The Rh gene of fish has been reported to be an intron-free gene [19]. In addition, we demonstrated that $\mathrm{cRh}-\mathrm{II} \mathrm{cDNA}$ had one Pst I site but that $\mathrm{cRh}$-I cDNA did not. Therefore, the $4 \mathrm{~kb}$ Pst I-digested band represented $\mathrm{cRh}-\mathrm{I}$ gene and the $2 \mathrm{~kb}$ Pst I-digested band represented cRh-II gene. Because both types of cRh cDNA did not have

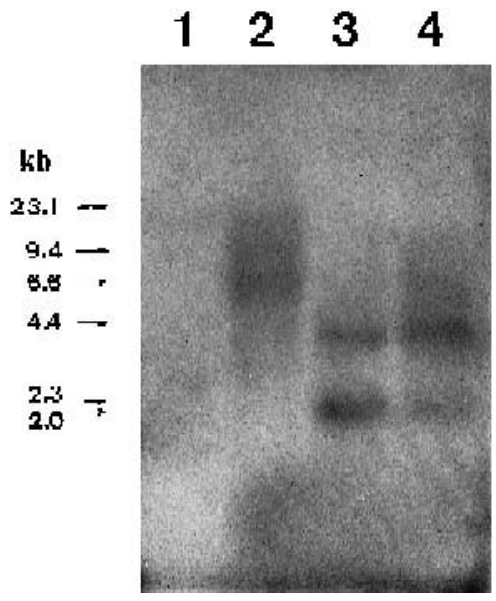

Fig. 2. Southern blot analysis of genomic DNA extracted from carp muscle. Genomic DNA $(10 \mu \mathrm{g})$ was digested with EcoRI (lane 2), Pst I (lane 3), and EcoRI + PstI (lane 4), respectively. After restriction, DNA fragments were resolved on the agarose gel, transferred into the membrane, and hybridized with a ${ }^{32} \mathrm{P}$ labelled cRh-I cDNA (nt 378-1136) probe in the solution of $5 \times$ Denhardt's, $50 \%$ formamide, $0.1 \mathrm{mg} / \mathrm{ml}$ calf thymus DNA, $0.1 \%$

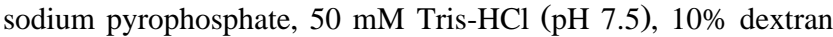
sulfate, $6 \times$ SSPE $\left(1 \times\right.$ SSPE: $0.15 \mathrm{M} \mathrm{NaCl}, 11 \mathrm{mM} \mathrm{NaH}_{2} \mathrm{PO}_{4}$, $1 \mathrm{mM}$ EDTA) and $0.5 \%$ SDS at $42^{\circ} \mathrm{C}$ overnight. The hybridized membrane was washed $4 \times, 3 \times$ and $2 \times$ SSC $(1 \times$ SSC: $0.15 \mathrm{M}$ $\mathrm{NaCl}$ and $15 \mathrm{mM}$ sodium citrate) with $10 \%$ SDS at $42^{\circ} \mathrm{C}$ for 30 min, respectively, before the membrane was exposed to the X-ray film for 9 days. The molecular marker, HindIII-digested lambda genome, was in lane 1 .

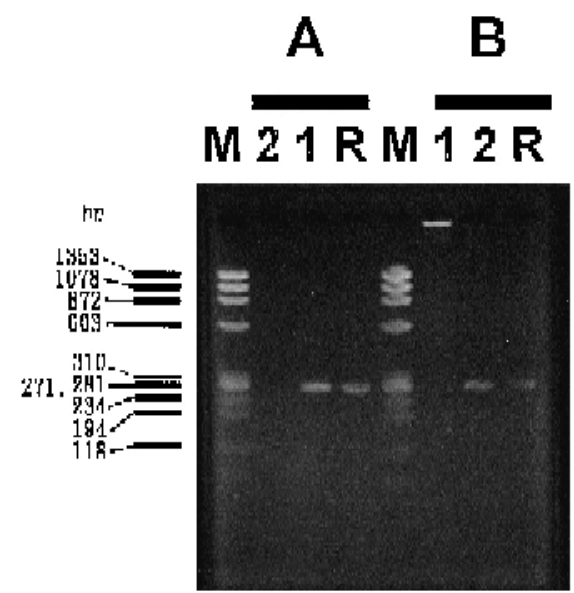

Fig. 3. RT-PCR analysis for the presence of cRh-I and cRh-II mRNA in carp retina. A specific primer set for type I (panel A) and for type II (panel B), respectively, were used to amplify the templates prepared from cRh-I cDNA (10 ng; lane I), cRh-II cDNA (10 ng; lane II), and total RNA (15 $\mu \mathrm{g}$; lane R) extracted from 10 pooled retinas of adult carp $(15-20 \mathrm{~cm}$ in length). The molecular marker, HindIII-digested lambda genome, was in lane M.

EcoRI sites, the $6.6 \mathrm{~kb}$ fragment from the EcoRI-digested genome included both cRh-I and cRh-II.

To determine if two rod opsin genes were expressed in carpretinas, we studied the total RNA extracted from adult carp using RT-PCR (Superscript $^{\mathrm{TM}}$ II RNase-H Reverse Transcriptase Kit, Gibco $\mathrm{BRL}$ ). Primers specific for $\mathrm{cRh}-\mathrm{I}$ mRNA were $5^{\prime}$-CCCCGGGAAAGAGCAACC-A-3' (nt 1221-1239; forward primer) and 5'-CATGCCTCTACAATCACAACAAA-3' (nt 1492-1470; reverse primer). Primers specific for cRh-II mRNA were 5'-CCAGGGAAACGAGCGACCG-3' (nt 1222-1240; forward primer) and 5'-CATGCCTCTAAAATCACAACAAC-3' (nt 1502-1480; reverse primer). A specific band, representing a mRNA fragment of expected size, showed up on the gel when the type-specific primer was used (Fig. 3). Clearly, both cRh-I and cRh-II gene were transcribed in carp retinas.

Comparison of the nucleotide sequences of the two types of $\mathrm{cRh}$, reveals that the region encoding the mature protein was conserved and the $3^{\prime}$ untranslated regions were particularly variable. In addition, Southern blot analysis of genomic DNA, and RT-PCR analysis of retinal RNA, indicate that the divergence of the $\mathrm{cRh}$ gene was not due to an in vitro artifact. 
The common carp, a cyprinid, is tetraploid. The presence of two rod opsins genes in the carp genome suggest that $\mathrm{cRh}-\mathrm{I}$ and $\mathrm{cRh}-\mathrm{II}$ originated from two different chromosome-pairs and have evolved independently. The closed-related goldfish has previously been shown to express two green cone opsin genes [6], although the extent of sequence divergence, at $8.5 \%$, is rather greater than that for the two rod opsins genes in carp. Therefore, genome duplication can explain the presence of two rod and green opsin genes in these fish.

We thank Dr. Kathryn Grant of the Department of Chemistry, Columbia University, NY, for providing pRJ1. This work was supported by a grant from the National Science Council, Republic of China (NSC85-2611-B-002-003).

\section{References}

[1] J.N. Lythgoe (1980) in: Environmental Physiology of Fishes (M.A. Ali, ed.), pp. 431-445, Plenum Press, New York.

[2] M. Yokote (1982) in: An Altas of Fish Histology (T. Hibiya, ed.), pp. 42-47, Gustav Fischer Verlag, Stuttgart.

[3] J. Nathans, D. Thomas, D.S. Hogness, Science 232 (1986) 193-202.

[4] F.I. Harosi, Y. Hashimoto, Science 222 (1983) 1021-1023.

[5] J.K. Bowmaker, Y.M. Kunz, Vision Res. 27 (1987) 21012108.

[6] R.L. Johnson, K.B. Grant, T.C. Zankel, M.F. Boehm, S.L.
Merbs, J. Nathans, K. Nakanishi, Biochemistry 32 (1993) $208-214$

[7] L. Stryer, J. Biol. Chem. 266 (1991) 10711-10714.

[8] H.G. Khorana, J. Biol. Chem. 267 (1992) 1-4.

[9] J. Nathans, D.S. Hogness, Cell 34 (1983) 807-814.

[10] J. Nathans, D.S. Hogness, Proc. Natl. Acad. Sci. USA 81 (1984) 4851-4855.

[11] C. Zuker, A.F. Cowman, G.M. Rubin, Cell 40 (1985) 851-858.

[12] M. Takao, A. Yasui, F. Tokunaga, Vision Res. 28 (1988) 471-480.

[13] M.R. Al-Ubaidi, S.J. Pittler, M.S. Champagne, J.T. Triantafyllos, J.F. McGinnis, W. Baehr, J. Biol. Chem. 265 (1990) 20563-20569.

[14] S.J. Pittler, S.J. Fliesler, W. Baehr, FEBS Lett. 313 (1992) $103-108$

[15] A. Morris, J.K. Bowmaker, D.M. Hunt, Proc. R. Soc. Lond. B 254 (1993) 233-240.

[16] O. Hisatomi, T. Iwasa, F. Tokunaga, A. Yasui, Biochem. Biophys. Res. Commun. 174 (1991) 1125-1132.

[17] S.S. Archer, J.N. Lythgoe, L. Hall, Proc. Royal Soc. Lond., Biol. Sci. B 248 (1992) 19-25.

[18] H.J. Tsai, S.R. Shih, C.M. Kuo, L.K. Li, Comp. Biochem. Physiol. 109B (1994) 81-88.

[19] J. Fitzgibbon, A. Hope, S.J. Slobodyanyuk, J. Bellingham, J.K. Bowmaker, D.M. Hunt, Gene 84 (1995) 273-277.

[20] F. Sanger, S. Nicklen, A.R. Coulson, Proc. Natl. Acad. Sci. USA 74 (1977) 5463-5467.

[21] T. Okano, D. Kojima, Y. Fukata, Y. Schichida, T. Yoshizawa, Proc. Natl. Acad. Sci. USA 89 (1992) 59325936.

[22] M.J.K. Rao, P. Argos, Biochim. Biophys. Acta 869 (1986) $197-214$ 